\title{
On the Level of Cluster Assembly Bias in SDSS
}

\author{
Ying $\mathrm{Zu}^{1,2 \star}$, Rachel Mandelbaum${ }^{2}$, Melanie Simet ${ }^{2}$, Eduardo Rozo ${ }^{3}$, Eli S. Rykoff ${ }^{4,5}$ \\ ${ }^{1}$ Center for Cosmology and AstroParticle Physics (CCAPP), Ohio State University, Columbus, OH 43210, USA \\ ${ }^{2}$ McWilliams Center for Cosmology, Department of Physics, Carnegie Mellon University, 5000 Forbes Avenue, Pittsburgh, PA 15213, USA \\ ${ }^{3}$ Department of Physics, University of Arizona, Tucson, AZ 85721, USA \\ ${ }^{4}$ Kavli Institute for Particle Astrophysics and Cosmology, Department of Physics, Stanford University, Stanford, CA 94305, USA \\ ${ }^{5}$ SLAC National Accelerator Laboratory, Menlo Park, CA 94025, USA
}

Accepted XXX. Received YYY; in original form ZZZ

\begin{abstract}
Recently, several studies have discovered a strong discrepancy between the large-scale clustering biases of two subsamples of galaxy clusters at the same halo mass, split by their average projected membership distances $\left\langle R_{\mathrm{mem}}\right\rangle$. The level of this discrepancy significantly exceeds the maximum halo assembly bias predicted by $\Lambda$ CDM. We explore whether some of the large-scale bias differences could be caused by projection effects in $\left\langle R_{\text {mem }}\right\rangle$ due to other systems along the line-of-sight. We thoroughly investigate the assembly bias of the redMaPPer clusters in SDSS, by defining a new variant of the average membership distance estimator $\tilde{R}_{\text {mem }}$ that is robust against projection effects in the cluster membership identification. Using the angular mark correlation functions, we show that the large-scale bias differences when splitting by $\left\langle R_{\text {mem }}\right\rangle$ can be mostly attributed to projection effects. After splitting by $\tilde{R}_{\text {mem }}$, the anomalously large signal is reduced, giving a ratio of $1.02 \pm 0.14$ between the two clustering biases as measured from weak lensing. Using a realistic mock cluster catalogue, we predict that the bias ratio between two $\tilde{R}_{\text {mem }}$-split subsamples should be $\simeq 1.10$, which is $>60 \%$ weaker than the maximum halo assembly bias (1.24) when split by halo concentration. Therefore, our results demonstrate that the level of halo assembly bias exhibited by clusters in SDSS is consistent with the $\Lambda$ CDM prediction. With a ten-fold increase in cluster numbers, deeper ongoing surveys will enable a more robust detection of halo assembly bias. Our findings also have important implications for quantifying the impact of projection effects on cosmological constraints using photometrically-selected clusters.
\end{abstract}

Key words: cosmology: observations - cosmology: large-scale structure of Universe — gravitational lensing: weak — methods: statistical

\section{INTRODUCTION}

The cold dark matter (CDM) structure formation theory predicts that the large-scale bias of halo clustering relative to the dark matter depends not only on halo mass, but also on other intrinsic halo properties such as concentration, formation time, substructure abundance, and spin (Sheth \& Tormen 2004; Gao et al. 2005; Wechsler et al. 2006; Harker et al. 2006; Jing et al. 2007; Li et al. 2008). In particular, above the characteristic non-linear mass scale, high-concentration halos have a lower clustering bias than their low-concentration counterparts, but for lower mass halos the trend is reversed - more concentrated halos exhibit higher clustering biases. This extra dependence of halo bias on properties other than halo mass, often referred to as "halo assembly bias" (Gao

^ E-mail: zu.4@osu.edu
\& White 2007), could be an important source of theoretical systematic uncertainty in the cosmological constraints (Wu et al. 2008; McEwen \& Weinberg 2016), and potentially leave imprints on the formation and distribution of galaxies (Berlind et al. 2006; Zhu et al. 2006; Weinmann et al. 2006; Blanton \& Berlind 2007; Croton et al. 2007; Zu et al. 2008; Deason et al. 2013; Kauffmann et al. 2013; Zentner et al. 2014; Lehmann et al. 2015; Paranjape et al. 2015; Zentner et al. 2016). Therefore, it is a vital task to directly detect halo assembly bias in observations and explore whether the observed assembly bias signal is consistent with theoretical expectations from $\Lambda \mathrm{CDM}$.

The assembly bias phenomena in low and high-mass regimes have very distinct theoretical origins. For low-mass halos, the assembly bias effect is mainly caused by the tidal heating and stripping of old halos in dense environments or by nearby larger systems, which suppressed the growth 
they would have otherwise experienced in the field (Diemand et al. 2007; Hahn et al. 2009). Additionally, when a bound group of subhalos was tidally disrupted after entering into a massive halo, a fraction of the subhalos would be ejected in highly-eccentric orbits and contribute to the overall assembly bias after they became distinct halos (Ludlow et al. 2009; Wang et al. 2009). For the very massive halos that we will focus on in this paper, Dalal et al. (2008) demonstrated that their assembly bias is directly related to the curvatures of Lagrangian peaks in the initial Gaussian random density field, analogous to the connection between linear halo bias and peak height in the peak background-split formalism (Bardeen et al. 1986; Sheth \& Tormen 1999). In a nutshell, two rare peaks of the same height but different curvatures usually appeared in different large-scale environments, and subsequently collapsed into two equal-mass clusters with different concentrations.

Detecting halo assembly bias signal in observations requires a robust halo finder, accurate measurements of halo mass, and a good proxy for halo concentration or formation time (see also Medezinski et al. 2016, for a novel experiment using the central entropies of X-ray emitting gas). For typical galaxy groups with $M_{h}<10^{13} h^{-1} M_{\odot}$ observed in the Sloan Digital Sky Survey (SDSS; York et al. 2000), most halo finders cannot robustly separate central galaxies from satellites, or identify which of two nearby groups hosts a given satellite (Campbell et al. 2015). Therefore, despite the fact that halo assembly bias is predicted to be stronger at lower mass, its observational signature in SDSS groups (Yang et al. 2007) has remained elusive to various detection efforts (Yang et al. 2006; Wang et al. 2013; Lacerna et al. 2014; Lin et al. 2016).

Recently, Miyatake et al. (2016, hereafter M16) have discovered a strong halo assembly bias effect among massive clusters $\left(\left\langle M_{200 m}\right\rangle \simeq 1.9 \times 10^{14} h^{-1} M_{\odot}\right)$ using the redMaPPer cluster catalogue (Rykoff et al. 2014) derived from SDSS Data Release 8 (DR8; Aihara et al. 2011). They split the clusters into two subsamples based on $\left\langle R_{\mathrm{mem}}\right\rangle$, the average projected distance of cluster membership candidates to the central galaxy, expecting the two sets of clusters to have different average halo concentrations. Using weak lensing, M16 discovered that the ratio between the large-scale clustering biases of the two subsamples is $\sim 1.64_{-0.26}^{+0.31}$, a $2.5 \sigma$ deviation from unity. Using the same cluster subsamples as M16, More et al. (2016) measured their cross-correlations with the SDSS photometric galaxy catalogue, and derived a much tighter constraint on the bias ratio $(1.48 \pm 0.07)$, which is $6.6 \sigma$ above unity but still consistent with M16. By adopting a linear model for the dependence of cluster bias on $\left\langle R_{\text {mem }}\right\rangle$, Baxter et al. (2016) found that a strong positive slope is required to fit the angular clustering of redMaPPer clusters split by $\left\langle R_{\mathrm{mem}}\right\rangle$, confirming the results of M16 and More et al. (2016).

Intriguingly, the bias ratios measured by those studies with the M16 estimate of $\left\langle R_{\mathrm{mem}}\right\rangle$ also exceed the level of halo assembly bias expected for similar clusters in the $\Lambda \mathrm{CDM}$. In particular, cosmological $\Lambda \mathrm{CDM}$ simulations predict that for a sample of massive haloes thresholded by the same comoving number density as the redMaPPer catalogue, the bias ratio between two subsamples split by their dark matter concentrations is $\simeq 1.24$, modulo minor variations due to uncertainties in cosmological parameters. Observationally, since $\left\langle R_{\mathrm{mem}}\right\rangle$ is usually estimated from the projected dis- tances of relatively bright satellites ( $~ 30$ per cluster), it is likely a much cruder indicator for assembly bias than the halo concentration measured in 3D from dark matter particles in simulations, even if the underlying satellite galaxy concentration somehow correlates better with halo assembly history than the dark matter concentration. Therefore, we consider 1.24 to be an upper limit of any observable level of cluster assembly bias using $\left\langle R_{\mathrm{mem}}\right\rangle$, yet the M16 and More et al. (2016) measurements exceed this maximum value by $1.5 \sigma$ and $3.4 \sigma$, respectively.

Without resorting to some new exotic physics, we are basically left with two possible observational explanations. The first is that the bias ratio anomaly could be merely a statistical fluke, which would disappear when a much larger cluster sample becomes available (Dalal 2016). On the other hand, there could be some systematic uncertainties that are unaccounted for in the estimate of $\left\langle R_{\mathrm{mem}}\right\rangle$, giving rise to a high bias ratio that is nonetheless irrelevant to halo assembly bias. In this paper, we examine the potential systematic uncertainties associated with the estimate of $\left\langle R_{\mathrm{mem}}\right\rangle$, particularly the impact of projection effects due to having multiple clusters on the same line-of-sight, and re-analyze the halo assembly bias signal within redMaPPer using a new $\left\langle R_{\text {mem }}\right\rangle$ estimator that is robust to such projections.

We organize the paper as follows. In $\S 2$ we investigate the possible imprint of projection effects on the distribution of membership probabilities in redMaPPer. We present a null test diagnostic for projection effects and develop a new $\left\langle R_{\text {mem }}\right\rangle$ estimator that passes this test in $\S 3$. In $\S 4$ we predict the level of observable assembly bias in redMaPPer after accounting for the scatter between $\left\langle R_{\mathrm{mem}}\right\rangle$ and halo concentration using mock cluster catalogues, and compare the prediction to the signal measured in redMaPPer from weak lensing. Finally, we summarize our findings and discuss their implications for future surveys in $\S 5$.

\section{PROJECTION EFFECT ON MEMBERSHIP PROBABILITIES}

Due to the lack of accurate distances, photometric clusterfinders suffer from various types of projection effects. In this paper, "projection effect" refers to the contamination of cluster membership probabilities by other systems along the same line-of-sight but outside the virial radius of that cluster. We emphasize that this particular effect is different from the common perception of projection in detecting clusters, i.e., the blending of multiple systems along the same line-of-sight into one large cluster (Erickson et al. 2011; Noh \& Cohn 2012). In the case of redMaPPer (Rykoff et al. 2014), there are two steps designed to remove the impact of projection effects on the estimate of the cluster richness $\lambda$ (for an alternative scheme see Castignani \& Benoist 2016):

- The member galaxy candidates of each cluster are searched within some finite aperture with a physical radius $R_{\lambda}$ that depends on the richness of the cluster $\lambda$,

$R_{\lambda} \simeq(\lambda / 100)^{0.2} \mathrm{Mpc} / h$,

so that all the member candidates are found within $R_{\lambda}$ of the cluster center and are assigned a membership probability $p_{\text {mem. }}$. 

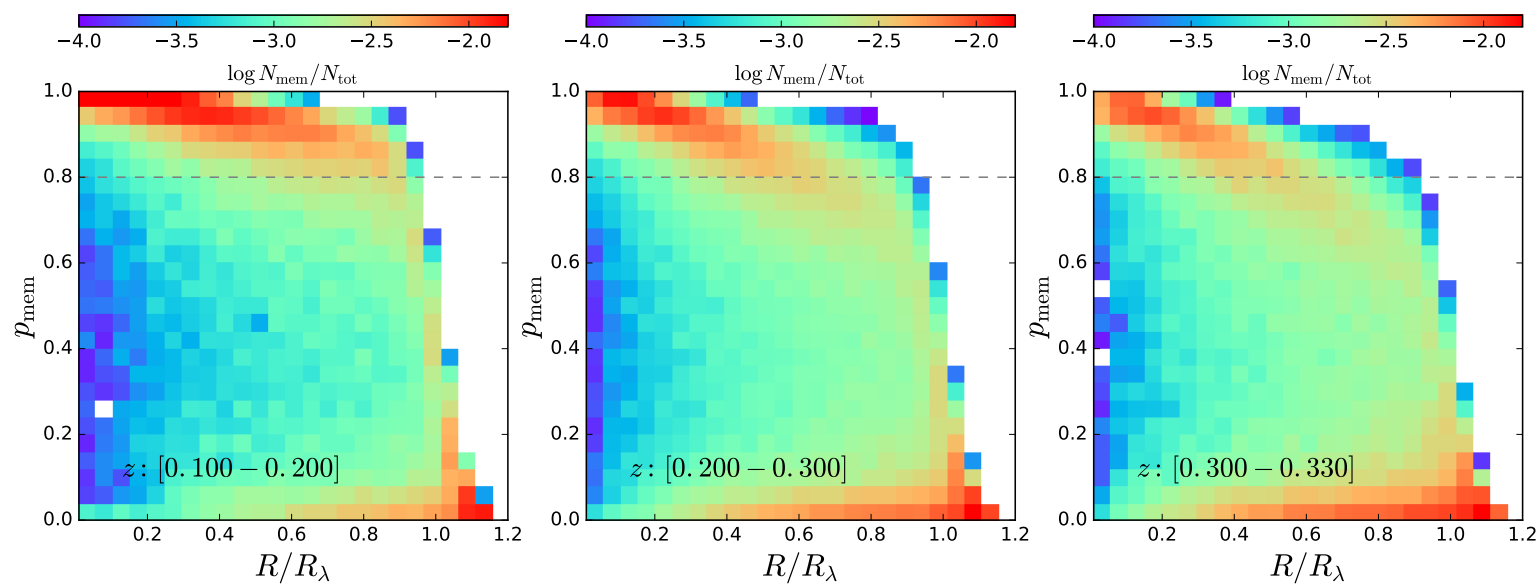

Figure 1. Member galaxy number distributions on the membership probability vs. projected distance (normalized by $R_{\lambda}$ defined by Equation 1) plane, for clusters within $z=[0.10,0.20]$ (left), $[0.20,0.30]$ (middle), and $[0.30,0.33]$, respectively. In each panel, the 2D distribution of logarithmic galaxy numbers (normalized by total number of galaxies) is color-coded by the color bar on the top. The low- $p_{\text {mem }}$ galaxies become more abundant and reach lower $\left\langle R_{\text {mem }}\right\rangle$ with increasing redshift. The horizontal line indicates the minimum $p_{m}=0.8$ that we used to define $\left\langle R_{\mathrm{mem}}^{\text {cut }}\right\rangle$.

- A percolation procedure was serially applied to all the clusters, assigning each candidate member galaxy a probability $p_{\text {free }}$ to account for the possibility of it being already assigned to another cluster along the same line-of-sight.

In addition, there are two other probabilities that describe the soft cuts in radius $\left(\Theta_{R}\right)$ and magnitude $\left(\Theta_{I}\right)$. Therefore, each galaxy within the aperture will have an effective membership probability $p_{m} \equiv p_{\text {mem }} \times p_{\text {free }} \times \Theta_{R} \times \Theta_{I}$. Note that although M16 used $p_{\text {mem }} \times p_{\text {free }}$ as the effective membership probability when estimating $\left\langle R_{\mathrm{mem}}^{\mathrm{M} 16}\right\rangle$, their large vs. small$\left\langle R_{\mathrm{mem}}^{\mathrm{M} 16}\right\rangle$ split is unchanged when $p_{m}$ is adopted. The galaxy surface number density profile of a cluster can then be estimated as

$\Sigma_{g}(R)=\frac{1}{2 \pi R \Delta R} \sum_{j} p_{m}^{j} \quad$ for $R_{j} \in R \pm \Delta R / 2$,

and the cluster richness is

$\lambda=\sum_{i} p_{m}^{i}=2 \pi \int_{0}^{R_{0}} R \Sigma_{g}(R) \mathrm{d} R$

where the index $i$ runs over all the membership galaxy candidates within the aperture.

Meanwhile, to compute $\left\langle R_{\mathrm{mem}}\right\rangle$, M16 applied the same membership weights to the projected distances

$\left\langle R_{\mathrm{mem}}^{\mathrm{M} 16}\right\rangle=\frac{\sum_{i}\left(p_{m}^{i} R_{i}\right)}{\lambda}=\frac{2 \pi}{\lambda} \int_{0}^{R_{0}} R^{2} \Sigma_{g}(R) \mathrm{d} R$.

In the case that the $p_{m}^{i}$ values are unbiased, $\left\langle R_{\mathrm{mem}}^{\mathrm{M} 16}\right\rangle$ is the correct estimator for $\left\langle R_{\mathrm{mem}}\right\rangle$. In cluster finders, the membership probability $p_{m}$ is closely tied to the expected density contrast between cluster members and background galaxies. In particular, the current version of the redMaPPer algorithm models the galaxy distribution surrounding the center of each cluster as the sum of an intrinsic cluster member component and a uniform background component, and then derives membership probabilities that are consistent with this two-component model (Rykoff et al. 2014). However, the background galaxy portion of the model is determined globally across the whole survey, and the $p_{m}$ assignment may thus be affected by fluctuations in the local density of background galaxies around individual clusters.

For instance, if the cluster were observed in a very crowded area on the sky, it is plausible that the cluster finder would incorrectly assign very low but non-zero values of $p_{m}$ to some background galaxies at large $R$, where the intrinsic galaxy number density profile $\Sigma_{g}(R)$ begins to drop precipitously. As a result, the observed $\Sigma_{g}(R)$ is more extended and flattened at large $R$, affecting the estimation of both $\lambda$ and $\left\langle R_{\mathrm{mem}}^{\mathrm{M} 16}\right\rangle$ simultaneously. To examine the impact of projection effects on the estimate of $\lambda$ in redMaPPer, Rykoff et al. (2014) performed a Monte Carlo test by randomly injecting simulated clusters with known $\lambda_{\text {true }}$ onto the actual observed background galaxy map, and measured the distribution of $\lambda_{\text {obs }}$ returned by redMaPPer at fixed $\lambda_{\text {true }}$. They found that most clusters fall within a tight locus around $\lambda_{\text {obs }} \simeq \lambda_{\text {true }}$, suggesting little systematic bias in the estimate of richness due to this global background model. However, comparing Equations 3 and 4, we can immediately tell that the estimation of $\left\langle R_{\mathrm{mem}}^{\mathrm{M} 16}\right\rangle$ is much more sensitive to the shape of $\Sigma_{g}(R)$ at large $R$ than that of $\lambda$. Therefore, even at fixed (or minimally biased) $\lambda$, the average membership distance estimated from Equation 4 could be systematically biased to larger values of $\left\langle R_{\mathrm{mem}}^{\mathrm{M} 16}\right\rangle$ in crowded areas than in isolated ones. Note that the contamination should persist in high- $p_{m}$ galaxies at some reduced level, but its impact on $\left\langle R_{\mathrm{mem}}^{\mathrm{M} 16}\right\rangle$ is likely much smaller.

Fig. 1 shows the member galaxy number distributions on the $2 \mathrm{D}$ plane of $p_{m}$ vs. $R / R_{\lambda}$, for clusters in redshift ranges $[0.1,0.2]$ (left), [0.2,0.3] (middle), and [0.3,0.33] (right), respectively. As expected, the average $p_{m}$ is a declining function of $R / R_{\lambda}$. More importantly, the distribution of $p_{m}$ at fixed $R / R_{\lambda}<0.9$ is bimodal, consisting of one population with extremely low values of $p_{m}$ and another with $p_{m}>0.6$. At large distances, the low- $p_{m}$ galaxies becomes dominant over the high- $p_{m}$ ones. This scale dependence of the relative fraction of the two populations should vary from cluster to cluster, depending on the level of contamination from local 


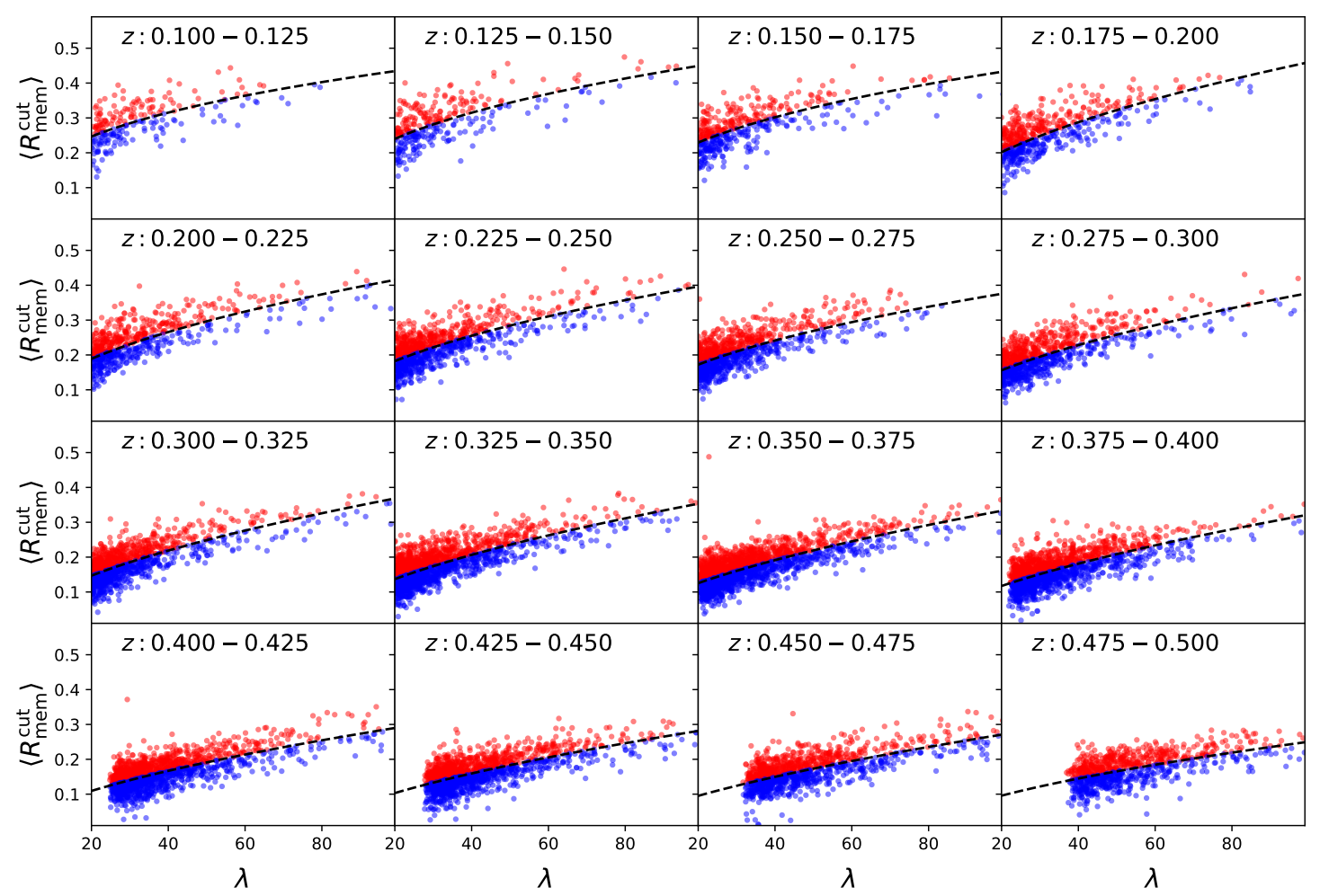

Figure 2. Subsamples of large (red) and small (blue) $\left\langle R_{\mathrm{mem}}^{\text {cut }}\right\rangle$ clusters on the $\left\langle R_{\mathrm{mem}}^{\text {cut }}\right\rangle$ vs. $\lambda$ plane at 16 different redshifts, using $p_{\mathrm{mem}}^{\text {crit }}=0.80$. The dashed curve in each panel indicates the median $\left\langle R_{\mathrm{mem}}^{\mathrm{cut}}\right\rangle$ as a function of richness at that redshift.

background. The low- $p_{m}$ population also becomes progressively more prominent with increasing redshift, while reaching further into the inner part of clusters. Therefore, placing a simple cut on $R / R_{\lambda}$ would not eliminate the low- $p_{m}$ galaxies at high redshifts. As described earlier, if the low $p_{m}$ values are more often incorrectly assigned in crowded regions on the sky, $\left\langle R_{\mathrm{mem}}^{\mathrm{M} 16}\right\rangle$ estimated from Equation 4 would be biased high for clusters in those regions.

There are two avenues for addressing the potential background contamination issue in membership assignments. One would be to improve the redMaPPer algorithm to account for the locally-varying background in determining $p_{m}$, in which case the $\left\langle R_{\mathrm{mem}}^{\mathrm{M} 16}\right\rangle$ estimator may be usable again. The alternative is to update the way we estimate $\left\langle R_{\mathrm{mem}}\right\rangle$. In this work we adopt the latter route as it can be trivially explored with the existing public redMaPPer catalogue.

In order to eliminate any problems caused by the contaminated low- $p_{m}$ galaxies, we define a variant of the average membership distance by placing a cut on $p_{m}$,

$\left\langle R_{\mathrm{mem}}^{\mathrm{cut}}\right\rangle=\frac{\sum_{i}\left(p_{m}^{i} R_{i}\right)}{\sum_{i} p_{m}^{i}} \quad$ for $p_{m}^{i}>p_{\mathrm{mem}}^{\mathrm{crit}}$

assuming the fractional amount of contamination decreases with increasing $p_{m}$. The choice of $p_{\text {mem }}^{\text {crit }}$ must satisfy two criteria:

- The projection effect is eliminated as determined using a suitable null test discussed below.

- The two cluster subsamples split by $\left\langle R_{\mathrm{mem}}^{\text {cut }}\right\rangle$ should ex- hibit different concentrations of their galaxy surface number density profiles.

After running both empirical tests (discussed further below) over a grid of $p_{m}$ cuts, we pick the value of $p_{\mathrm{mem}}^{\text {crit }}=0.8$, as shown by the dashed horizontal lines in Fig. 1. Under this cut, the effective richness, calculated by summing all the $p_{m}$ above $p_{\text {mem }}^{\text {crit }}$, shrinks significantly at high redshifts, introducing some extra scatter into the ranking-order of $\left\langle R_{\mathrm{mem}}\right\rangle$ at fixed $z$. However, even at the highest redshift bin $(z \sim 0.30)$ almost all $(>98 \%)$ the clusters still retain more than 6 member galaxy candidates for computing $\left\langle R_{\mathrm{mem}}^{\text {cut }}\right\rangle$, and the fraction (weighted by $p_{m}$ ) of all member galaxies with $p_{m}>0.8$ is $\sim 0.55$.

Fig. 2 illustrates the division of clusters into large and small- $\left\langle R_{\mathrm{mem}}^{\text {cut }}\right\rangle$ subsamples at 16 different redshifts bins from $z=0.1$ to 0.5 . At each redshift $z$, we separate the clusters by the median of their $\left\langle R_{\mathrm{mem}}^{\text {cut }}\right\rangle$ as a function of $\lambda$ (black dashed curve). Note that beyond $z=0.33$ the sample systematically misses low-richness clusters, as the luminosity threshold for membership galaxies $\left(0.2 L_{*}\right)$ hits the magnitude limit of the DR8 catalogue $(i<21)$. We nonetheless make use of all clusters up to $z=0.50$ for the null test of projection effects (discussed below in $\S 3$ ), which does not require samplecompleteness in richness. However, we will limit our assembly bias analysis (see $\S 4$ ) to clusters between 0.1 and 0.33 , where the redMaPPer catalogue is approximately volumecomplete.

Fig. 2 also shows that both the average amplitude and the scaling of median $\left\langle R_{\mathrm{mem}}^{\text {cut }}\right\rangle$ with $\lambda$ vary smoothly with 


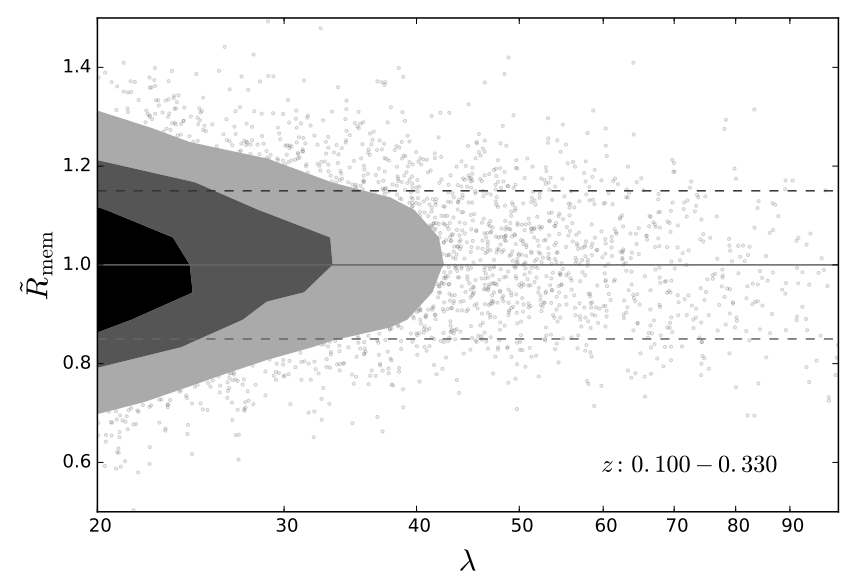

Figure 3. Distribution of the reduced average membership distance $\tilde{R}_{\text {mem }}$ measured as a function of cluster richness $\lambda$. The three contour lines enclose $25 \%, 50 \%$, and $75 \%$ of the clusters, respectively. Unlike the distributions of $\left\langle R_{\mathrm{mem}}^{\mathrm{cut}}\right\rangle$ shown in Figure. 2, the mean (1) and the standard deviation (0.15) of the distributions of $\tilde{R}_{\text {mem }}$ are uniform across the entire redshift range of the redMaPPer sample.

redshift. In order to quantify the deviations of individual $\left\langle R_{\text {mem }}^{\text {cut }}\right\rangle$ from the median and compare them across different $\lambda$ and $z$, we fit a power-law relation $\overline{\left\langle R_{\mathrm{mem}}^{\text {cut }}\right\rangle}(\lambda, z)=a(z) \lambda^{b(z)}$ to the median relation at each $z$, and spline-interpolate the best-fitting parameters $a(z)$ and $b(z)$ over the entire redshift range. In this way, we can classify a cluster with given $\left\langle R_{\mathrm{mem}}^{\text {cut }}\right\rangle$ into large or small- $\left\langle R_{\mathrm{mem}}^{\text {cut }}\right\rangle$ subsamples directly based on its $\lambda$ and $z$.

Furthermore, we normalize the deviations of $\left\langle R_{\mathrm{mem}}^{\text {cut }}\right\rangle$ from $\overline{\left\langle R_{\mathrm{mem}}^{\text {cut }}\right\rangle}(\lambda, z)$ by the standard deviation of $\left\langle R_{\mathrm{mem}}^{\text {cut }}\right\rangle$ at fixed $\lambda$ and $z$, i.e., defining a reduced average membership distance $\tilde{R}_{\mathrm{mem}}=1+0.15\left(\left\langle R_{\mathrm{mem}}^{\text {cut }}\right\rangle-\overline{\left\langle R_{\mathrm{mem}}^{\text {cut }}\right\rangle}(\lambda, z)\right) / \sigma_{\left\langle R_{\mathrm{mem}}^{\text {cut }}\right\rangle}(\lambda, z)$, so that the distribution of $\tilde{R}_{\text {mem }}$ always has a mean of unity and a dispersion of 0.15 (arbitrarily chosen). As a result, the probability distribution functions of $\tilde{R}_{\text {mem }}$ are almost identical across any fixed richness and redshift. Fig. 3 shows the cluster number distribution on the $\tilde{R}_{\text {mem }}$ vs. $\lambda$ plane, with two dashed horizontal lines indicating the $1-\sigma$ range of $\tilde{R}_{\text {mem }}$ at fixed $\lambda$. To facilitate the comparison between the M16 results and ours, we apply the same transformation to the distribution of $\left\langle R_{\mathrm{mem}}^{\mathrm{M} 16}\right\rangle$ to obtain $\tilde{R}_{\mathrm{mem}}^{\mathrm{M} 16}=1+0.15\left(\left\langle R_{\mathrm{mem}}^{\mathrm{M} 16}\right\rangle-\overline{\left\langle R_{\mathrm{mem}}^{\mathrm{M} 16}\right\rangle}(\lambda, z)\right) / \sigma_{\left\langle R_{\mathrm{mem}}^{\mathrm{M} 16}\right\rangle}(\lambda, z)$. By construction, the distribution of $\left\langle R_{\mathrm{mem}}^{\mathrm{M} 16}\right\rangle$ (not shown here) is similar to Fig. 3.

Fig. 4 provides a direct evidence of the impact of projection effects in the estimate of $\left\langle R_{\mathrm{mem}}^{\mathrm{M} 16}\right\rangle$. For a given cluster at $z \in[0.30,0.33],{ }^{1}$ we identify all the physically unassociated (explained further below) clusters that happen to lie along the same line-of-sight, and compute the fraction of

1 We pick this redshift bin for Fig. 4 and later the mark correlation functions because it shows the most prominent population of low- $p_{m}$ galaxies, and therefore is the most susceptible to projection effects.

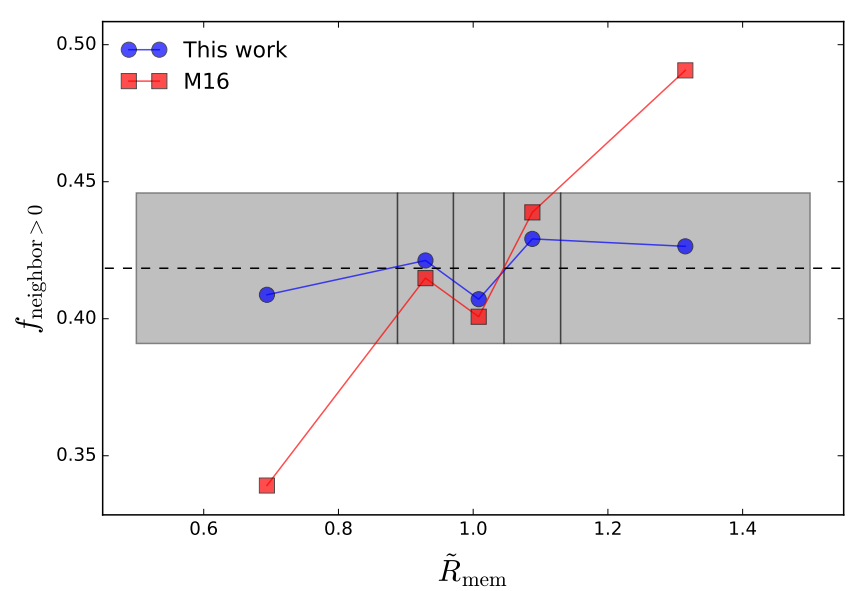

Figure 4. Fraction of clusters that have one or multiple physically unassociated clusters along the same line-of-sight as a function of $\tilde{R}_{\text {mem }}$ (blue circles) or $\tilde{R}_{\text {mem }}^{\mathrm{M} 16}$ (red squares). For a given cluster at $z \in[0.3,0.33]$, we count any other clusters that are observed at $\Delta z=0.02$ away and within a solid angle that corresponds to $3.2 h^{-1} \mathrm{Mpc}$ at $z$ as "physically unassociated neighbors". The gray band indicates the $1 \sigma$ uncertainty for a $\left\langle R_{\mathrm{mem}}\right\rangle$ estimator free of projection effects.

cluster sight-lines that have one or multiple such apparent neighbors as a function of $\tilde{R}_{\text {mem }}^{\mathrm{M} 16}$ (red squares) or $\tilde{R}_{\text {mem }}$ (blue circles). Specifically, we search secondary clusters within a solid angle that corresponds to $d=3.2 h^{-1} \mathrm{Mpc}$ at the redshift of the primary, and at a redshift at least $\Delta z=0.02$ away from the primary. This value of $\Delta z$ is $33 \%$ larger than the cluster photo-z uncertainty $\left(\sigma_{z}=0.015\right.$ at $z=0.3$; Rykoff et al. 2014), so that the two systems are unlikely to be physically associated. We have also verified that using different choices of $d \in[2,4]$ and $\Delta z \in[0.03,0.04]$ do not qualitatively change the result of this experiment. The gray band indicates the $1 \sigma$ uncertainty for a $\left\langle R_{\text {mem }}\right\rangle$ measure that is free of projection effects, derived from a Monte Carlo test in which we re-measure $f_{\text {neighbor }>0}$ after randomly shuffling the $\tilde{R}_{\mathrm{mem}}^{\mathrm{M} 16}$ values among clusters and repeat the exercise 50000 times. The uncertainties are identical across all five $\tilde{R}_{\text {mem }}^{\mathrm{M} 16}$ bins as there are equal numbers of clusters in each bin by design.

Clearly, if a cluster is detected with some other unassociated systems projected close to its sight-line, it is significantly more likely to have a higher estimated value of $\left\langle R_{\mathrm{mem}}^{\mathrm{M} 16}\right\rangle$ than one without. In contrast, our new $\left\langle R_{\mathrm{mem}}^{\mathrm{cut}}\right\rangle$ estimator is consistent with being free of such projection effects, showing a uniform $f_{\text {neighbor }>0}$ as a function of $\tilde{R}_{\text {mem }}$. Therefore, Fig. 4 confirms our expectation that the background contamination in low- $p_{m}$ galaxies causes systematic biases in the estimate of $\left\langle R_{\mathrm{mem}}^{\mathrm{M} 16}\right\rangle$, and by simply removing them when defining $\left\langle R_{\mathrm{mem}}^{\text {cut }}\right\rangle$ we can significantly reduce or even eliminate the impact of projection effects on $\left\langle R_{\mathrm{mem}}\right\rangle$.

However, one drawback of the $f_{\text {neighbor }}$ test in Fig. 4 is that, it probes only one distance scale $d$ each time, while the halo assembly bias exists on all scales above 1halo (Sunayama et al. 2016) and is usually measured on scales above $10 h^{-1} \mathrm{Mpc}$ - a non-parametric null test that can probe all scales is required. 


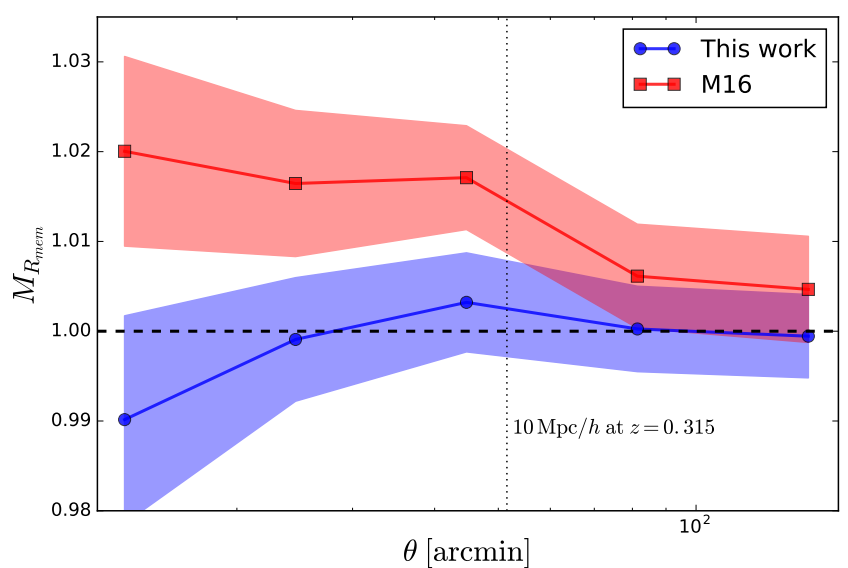

Figure 5. Angular mark cross-correlation functions between clusters at $z \in[0.30,0.33]$ and the joint cluster samples at $z \in$ $[0.10,0.28]$ and $z \in[0.35,0.50]$, using $\tilde{R}_{\text {mem }}^{\mathrm{M} 16}$ (red) and $\tilde{R}_{\text {mem }}$ (blue) as marks, respectively. The vertical dashed line indicates the angular distance that corresponds to a physical separation of $10 h^{-1} \mathrm{Mpc}$ at $z=0.3$

\section{A NULL TEST FOR DIAGNOSING PROJECTION EFFECTS}

We employ the angular mark cross-correlation function between two cluster samples that are well-separated in redshift as a null test for diagnosing projection effects in each $\left\langle R_{\text {mem }}\right\rangle$ definition. The angular mark correlation is defined as (Stoyan \& Stoyan 1994; Beisbart \& Kerscher 2000; Sheth et al. 2005; Skibba et al. 2006; Harker et al. 2006)

$M_{R_{\mathrm{mem}}}(\theta)=\frac{1+\mathcal{W}(\theta)}{1+w(\theta)}$,

where $\mathcal{W}$ and $w$ are the $\left\langle R_{\text {mem }}\right\rangle$-weighted and regular angular correlation functions, respectively, and $\theta$ is the angular distance on the sky. Within each cluster sample the marks $\tilde{R}_{\mathrm{mem}}^{\mathrm{M} 16}$ and $\tilde{R}_{\text {mem }}$ are normalized to have a mean of unity. Therefore, if the marks of a pair of clusters from two different samples separated by distance $\theta$ do not correlate with each other, the mark correlation $M$ would be exactly unity at $\theta$. In practice the angular mark correlation function can be directly computed via dividing the mark-weighted pair counts $W W$ by the unweighted ones $D D$

$M_{R_{\mathrm{mem}}}(\theta)=\frac{W W(\theta)}{D D(\theta)}$,

thus avoiding the need of random catalogues. Compared to the $f_{\text {neighbor }}$ test in Fig. $4, M_{R_{\mathrm{mem}}}(\theta)$ is an indirect but statistically more powerful null test of projection effects, as it probes all angular scales at once by using all cluster pairs in the catalogue.

Fig. 5 shows the angular mark correlation functions between clusters with $z \in[0.30,0.33]$ and all other clusters with either $z \in[0.10,0.28]$ or $z \in[0.35,0.50]$ in redMaPPer, using $\tilde{R}_{\text {mem }}^{\mathrm{M} 16}(\mathrm{red})$ and $\tilde{R}_{\text {mem }}$ (blue) as markers, respectively. Using a large-volume $N$-body simulation $\left(2.5^{3} h^{-3} \mathrm{Gpc}^{3}\right.$; described further below), we have verified that the redshift separation between the slices, $\Delta z=0.02$, which corresponds to a comoving distance of $52 h^{-1} \mathrm{Mpc}$ at $z=0.3$, is large enough so that

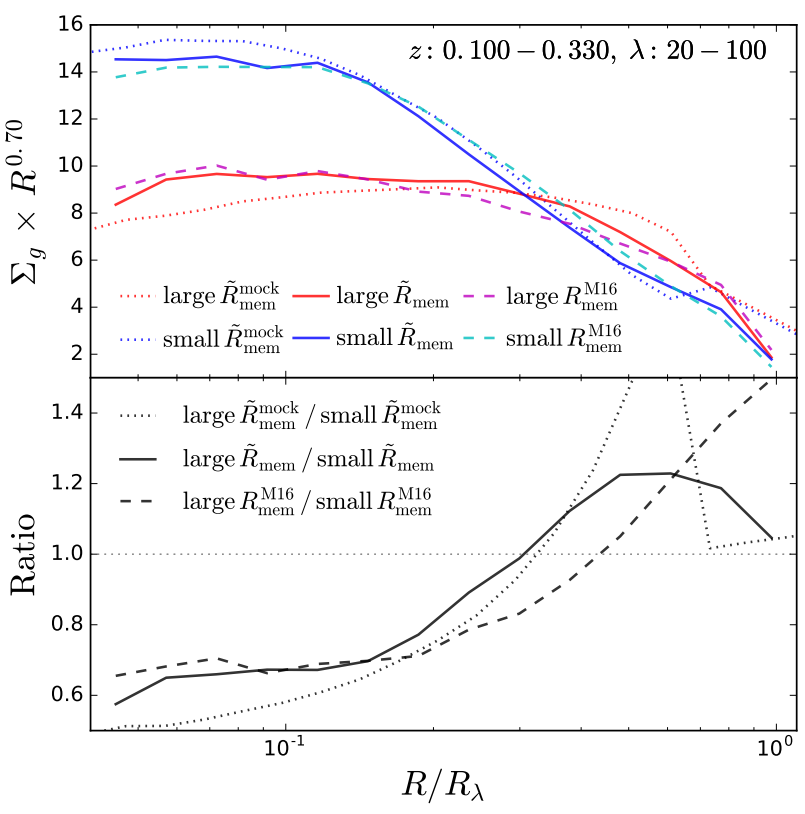

Figure 6. Member galaxy surface density profiles $\Sigma_{g}$ (with $p_{m}$ weighting) of the large (red or magenta) and small (blue or cyan)$\left\langle R_{\text {mem }}\right\rangle$ clusters, split by $\tilde{R}_{\text {mem }}^{\mathrm{M} 16}$ (dashed) and $\tilde{R}_{\text {mem }}$ (solid), respectively. The dotted curves are the surface density profiles predicted by a mock cluster catalogue that mimics the $\left\langle R_{\text {mem }}^{\text {cut }}\right\rangle$-split. The bottom panel shows the ratio between the $\Sigma_{g}(R)$ of high and lowconcentration clusters within each definition of $\left\langle R_{\mathrm{mem}}\right\rangle$. The mock cluster catalogue correctly reproduces the contrast between the large and small- $\left\langle R_{\mathrm{mem}}^{\mathrm{cut}}\right\rangle$ clusters (compare solid to dotted curves), especially the distance at which the ratio crosses unity in the bottom panel.

the mark correlation function (using halo concentrations as markers) is consistent with unity, i.e., there is little physical assembly bias left.

This angular marked statistics $M_{\left\langle R_{\mathrm{mem}}\right\rangle}$ serves as our null test for the presence of projection effects in any estimators of $\left\langle R_{\mathrm{mem}}\right\rangle$ : for a $\left\langle R_{\mathrm{mem}}\right\rangle$ estimator free of membership contaminations, its $M_{\left\langle R_{\mathrm{mem}}\right\rangle}$ should be unity on all angular scales because two cluster samples in the same sky area but different redshift ranges are not physically associated. Clearly, our new estimator $\tilde{R}_{\text {mem }}$ passes this null test on all measured scales $\in[10,200]$ arcmin, while the M16 estimator $\left\langle R_{\mathrm{mem}}^{\mathrm{M} 16}\right\rangle$ does not, showing spatially coherent and statistically significant correlation between pairs of physically unassociated clusters on the sky.

Combining Figs. 4 and 5, we establish that the $\left\langle R_{\mathrm{mem}}^{\mathrm{M} 16}\right\rangle$ selection is affected by projection effects that have contaminated the photometric galaxy membership probabilities at low values of $p_{m}$, while the $\left\langle R_{\mathrm{mem}}^{\text {cut }}\right\rangle$ selection is almost immune to such projection effect, by separating high and lowconcentration clusters based only on member galaxies with high $p_{m}$.

One might worry that the subsamples split by $\left\langle R_{\mathrm{mem}}^{\text {cut }}\right\rangle$, which uses only $\sim 55 \%$ of the total membership candidates, do not have average mass/galaxy density profiles with distinct concentrations. To find out, we compare the galaxy surface number density profiles of large and small- $\left\langle R_{\text {mem }}\right\rangle$ clus- 
ters in Fig. 6, split by $\left\langle R_{\mathrm{mem}}^{\mathrm{M} 16}\right\rangle$ (dashed) and $\left\langle R_{\mathrm{mem}}^{\text {cut }}\right\rangle$ (solid), separately. The $\Sigma_{g}$ profiles are computed using Equation 2 with the $p_{m}$-weighting on each galaxy. Also note that when computing the $\Sigma_{g}$ profiles for $\tilde{R}_{\text {mem-selected clusters, we do }}$ not place any cut on $p_{m}$ and employ all member candidates with $p_{m}>0$. Both ways of splitting samples return two sets of high and low-concentration $\Sigma_{g}$ profiles, with similar amplitudes at small $R$.

The dashed curves in Fig. 6 are the measurements from a mock cluster catalogue and the $R_{\text {mem }}^{\text {mock }}$ estimator is designed to mimic the behavior of $\left\langle R_{\mathrm{mem}}^{\text {cut }}\right\rangle$ in redMaPPer (see $\S 4$ ). We will describe the detail of this mock cluster catalogue and the definition of $R_{\text {mem }}^{\text {mock }}$ in the section below, and then come back to Fig. 6 .

\section{A RE-ANALYSIS OF CLUSTER ASSEMBLY BIAS}

As alluded to in $\S 1$, the cluster assembly bias signal revealed by $\left\langle R_{\text {mem }}\right\rangle$ in observations would likely be diluted compared to the signal predicted from simulations using dark matter concentration $(c)$, due to the large scatter of $\left\langle R_{\text {mem }}\right\rangle$ at fixed $c$. In the case of $\left\langle R_{\text {mem }}^{\text {cut }}\right\rangle$, the scatter $\sigma_{\left\langle R_{\text {mem }}^{\text {cut }}\right\rangle \mid c}$ for any given cluster mass has three contributing sources:

1) The intrinsic spread in the distribution of the underlying galaxy concentration $c_{g}$ at fixed $c$.

2) The observational scatter between $\left\langle R_{\mathrm{mem}}^{\text {cut }}\right\rangle$ and $c_{g}$ within clusters, due to Poisson fluctuations in the galaxy number density profile, the use of projected distances (instead of radial distances), and the small aperture size for selecting members.

3) The systematic uncertainty in $\left\langle R_{\mathrm{mem}}^{\text {cut }}\right\rangle$ due to some residual contamination in the membership probabilities of the $p_{m}>0.8$ galaxies.

Among the above three, we expect the observational scatter between $\left\langle R_{\mathrm{mem}}^{\text {cut }}\right\rangle$ and $c_{g}$ to be the dominant source of signal dilution. For the spread in the distribution of $c_{g}$ at fixed $c$, it depends on how well the satellite galaxies follow the orbits, and hence the spatial distribution, of dark matter particles. Although the radial distribution of subhalos at or above some fixed present-day mass $m_{0}$ is flatter than the density profile of the host halo (Diemand et al. 2004; Gao et al. 2004), the radial profile of subhalos selected on their tidally truncated mass (or the peak mass over their assembly history $m_{\text {peak }}$ ) traces the dark matter very well, except at the inner radius where dynamical friction would affect the distribution of more massive subhalos (Nagai \& Kravtsov 2005; Han et al. 2016; van den Bosch et al. 2016). Since we expect galaxies selected on stellar mass or luminosity correspond closely to subhalos selected on $m_{\text {peak }}$ rather than $m_{0}$, it is reasonable to expect the intrinsic scatter in $c_{g}$ at fixed $c$ to be subdominant compared to the observational scatter in $\left\langle R_{\mathrm{mem}}^{\text {cut }}\right\rangle$ at fixed $c_{g}$. For the residual systematic uncertainties in $\left\langle R_{\mathrm{mem}}^{\text {cut }}\right\rangle$, we expect it to be negligible at the noise level of redMaPPer, based on the null test in Figure 5. The mark correlation has a slight dip below unity in the lowest angular distance bin at 15 arcmin, but it is more likely a statistical fluctuation within the uncertainty and should not be caused by projection effects, which only leads to correlations above unity.
Therefore, before measuring the assembly bias signal using $\left\langle R_{\mathrm{mem}}^{\mathrm{cut}}\right\rangle$ in redMaPPer, we need to carefully examine the impact of the scatter between $\left\langle R_{\mathrm{mem}}^{\text {cut }}\right\rangle$ and $c_{g}$ on the observable level of signal, using mock catalogues of massive clusters and red-sequence galaxies. For the mock cluster catalogue, we make use of the ROCKSTAR (Behroozi et al. 2013) halo catalogue derived from the $z=0.25$ output of the BigMDPL simulation ${ }^{2}$. The simulation was evolved under Planck cosmology (Planck Collaboration et al. 2015) with $3840^{3}$ particles in a cubic box of $2.5 h^{-1} \mathrm{Gpc}$ on a side (Klypin et al. 2016). The large box size is necessary to ensure that the large-scale bias measurements are not limited by cosmic variance. We describe the construction of mock cluster and red-sequence galaxy catalogues in detail below.

We employ the iHOD framework recently developed by Zu \& Mandelbaum $(2015,2016,2017)$ to populate halos with mock galaxies that reproduce the low-redshift lensing and clustering measurements of SDSS galaxies, and from them we further select a red-sequence population based on the halo-quenching model of Zu \& Mandelbaum (2016). Similarly, the stellar mass and color distributions of the mock galaxies closely mimic the observed galaxies at $z=0.25(\mathrm{Zu}$ \& Mandelbaum 2017), because the best-fitting parameters for the iHOD halo-quenching prescription are derived from the spatial clustering and galaxy-galaxy lensing measurements of SDSS galaxies at $z \in[0.0,0.3]$.

Within each halo, most of the subhalos are not wellresolved in BigMDPL due to its relatively low mass resolution $\left(m_{p}=2.34 \times 10^{10} h^{-1} M_{\odot}\right)$. Therefore, instead of us-

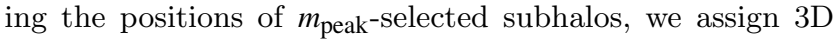
distances to satellite galaxies based on an NFW profile with galaxy concentration $c_{g}=0.86 c$. The slightly underconcentrated galaxy distribution is preferred by the smallscale clustering and $\mathrm{g}-\mathrm{g}$ lensing measurements of SDSS galaxies (Zu \& Mandelbaum 2015) and the observed radial distribution of satellites inside clusters (Yang et al. 2005; Chen et al. 2006; Budzynski et al. 2012; Wang et al. 2014; Zenteno et al. 2016).

The richness of each mock cluster is assigned by counting the number of red galaxies within $r_{200 \mathrm{~m}}$ and above the stellar mass threshold $M_{*}^{\lim }=1.66 \times 10^{10} h^{-2} M_{\odot}$. We pick this value of $M^{\text {lim }}$ so that the mean halo mass of our mock clusters with $\lambda$ between 20 and 100 is the same as in the redMaPPer catalogue $\left(\left\langle M_{h}\right\rangle \simeq 1.86 \times 10^{14} h^{-1} M_{\odot}\right.$; see Miyatake et al. 2016; Simet et al. 2016). Note that the richness defined in our mock uses a different aperture than in redMaPPer, where the richness is derived using the richness-dependent aperture $R_{\lambda}$ via a complicated iterative scheme. This difference, however, does not affect the comparison between the mock and redMaPPer cluster samples, as the mock richness is merely used for selecting clusters, and we are not comparing the two on individual cluster basis.

Finally, we need to incorporate the small aperture effect in the $\left\langle R_{\mathrm{mem}}^{\text {cut }}\right\rangle$ estimator due to the $p_{m}>0.8$ selection (see Fig. 1). Ideally, we would want to simulate the $p_{m}$ values first by running the redMaPPer cluster finder over a mock SDSS imaging catalogue, and select only high- $p_{m}$ mock galaxies when deriving $\left\langle R_{\mathrm{mem}}^{\text {cut }}\right\rangle$ for the detected mock clusters. However, this is a rather difficult task, requiring a near-

\footnotetext{
2 https://www.cosmosim.org/cms/simulations/bigmdpl
} 
perfect understanding of the photometric redshift properties of SDSS galaxies up to $z=0.55$. In reality, since the amount of background contamination in $\left\langle R_{\mathrm{mem}}^{\text {cut }}\right\rangle$ is negligible, the effective aperture size associated with the $p_{m}>0.8$ selection should not depend on the local background. Therefore, we can bypass the task of simulating realistic $p_{m}$, and assume the small aperture effect due to a cut in $p_{m}$ can be roughly mimicked by adopting an effective aperture size that is some fixed fraction $a$ of $R_{\lambda}$ in the mock. We empirically determine the value of $a$ to be 0.65 , by enforcing that the fraction of $R<R_{\lambda}$ galaxies included by this $R<a R_{\lambda}$ cut is 0.55 , the same as that included by the $p_{m}>0.8$ cut in redMaPPer. For each mock cluster, we now define $\left\langle R_{\text {mem }}^{\text {mock }}\right\rangle$ as the average $2 \mathrm{D}$ membership distance of galaxies within $0.65 R_{\lambda}$.

To check whether the scatter between $\left\langle R_{\text {mem }}^{\text {mock }}\right\rangle$ and $c_{g}$ is comparable to the intrinsic scatter between $\left\langle R_{\mathrm{mem}}^{\text {cut }}\right\rangle$ and $c_{g}$ within redMaPPer, we now go back to the mock vs. redMaPPer galaxy surface density profiles shown in Fig. 6 . In the top panel, the mock galaxy density profiles of the large and small- $\left\langle R_{\text {mem }}^{\text {mock }}\right\rangle$ clusters (dotted) roughly reproduce both the shapes and amplitudes of the real $\left\langle R_{\mathrm{mem}}^{\text {cut }}\right\rangle$-split cluster profiles (solid). If the scatter in the mock is much larger (smaller) than in the observations, the contrast between the galaxy surface density profiles of the two subsamples would be much lower (higher). The ratio between the $\left\langle R_{\mathrm{mem}}^{\text {mock }}\right\rangle$-split profiles in the mock $(0.60)$ is slightly lower than that between the $\left\langle R_{\mathrm{mem}}^{\text {cut }}\right\rangle$-split profiles in redMaPPer $(0.65)$ at $R / R_{\lambda} \sim 0.1$ (dotted vs. solid curves in the lower panel), suggesting a similar but slightly under-estimated scatter in the mock compared to the data. Furthermore, the distance at which the ratio of two $R_{\text {mem }}^{\text {mock }}$-split profiles crosses unity is $\simeq 0.3 R_{\lambda}$, in excellent agreement with that of the $\left\langle R_{\mathrm{mem}}^{\text {cut }}\right\rangle$-split samples. This agreement indicates that the $0.65 R_{\lambda}$ aperture we adopted for $\left\langle R_{\text {mem }}^{\text {mock }}\right\rangle$ is close to the effective aperture of $\left\langle R_{\mathrm{mem}}^{\text {cut }}\right\rangle$ in redMaPPer. Note that since redMaPPer used a 2D aperture $R_{\lambda}$ instead of $r_{200 m}$ to derive $\lambda$, we boost the galaxy surface number density profiles of the mock clusters by $\lambda / N_{R_{\lambda}}$ to match to observations in Fig. 6, where $\lambda$ and $N_{R_{\lambda}}$ are the richness calculated from including all mock galaxies within $r_{200 m}$ and the number of mock galaxies within a cylinder of radius $R_{\lambda}$ (still within a sphere of $\left.r_{200 m}\right)$, respectively.

With both the mock cluster and membership galaxy catalogues at hand, we first measure several different types of $\left\langle R_{\mathrm{mem}}\right\rangle$, and compare them with the underlying dark matter concentration in Fig. 7. From top to bottom, the four contours show the distributions of four different types of $\left\langle R_{\text {mem }}\right\rangle$ against concentration $c$, including 1$)$ the 3D average membership distance averaged over all members within $\left.r_{200 m}, 2\right) 2 \mathrm{D}$ average membership distance averaged over all members within $\left.r_{200 m}, 3\right) 2 \mathrm{D}$ average membership distance averaged over all members within $R_{\lambda}$, and 4) 2D average membership distance averaged over all members within $R_{\lambda}$, for mock clusters with richness between 32 and 34 (the average richness of the sample is 33). The horizontal dotted lines indicate the median values of $\left\langle R_{\mathrm{mem}}\right\rangle$ for the four estimators, and the vertical dotted line the median value of halo concentration. Unsurprisingly, the $3 \mathrm{D}\left\langle R_{\mathrm{mem}}\right\rangle$ estimator (gray) shows the strongest correlation with $c$, with a Spearman's cross-correlation coefficient of $\rho_{c c}=-0.48$, where the scatter is entirely due to stochasticity of small galaxy numbers per cluster. The correlation becomes slightly weaker when

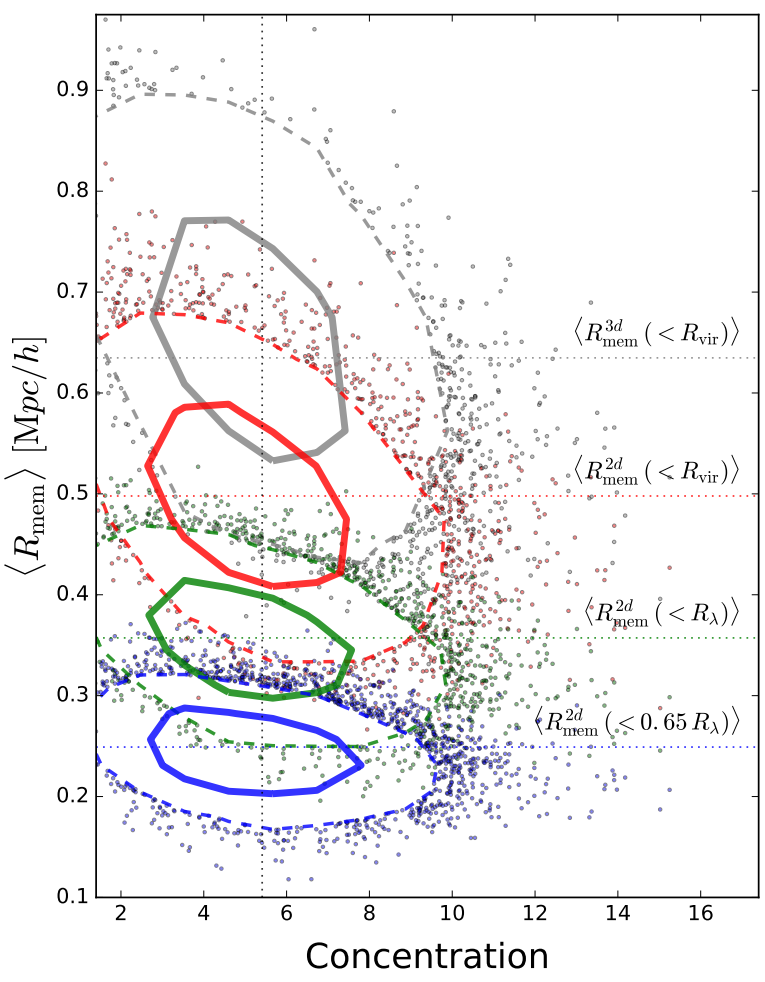

Figure 7. Comparison between different choices of $\left\langle\boldsymbol{R}_{\mathrm{mem}}\right\rangle$ as proxies of halo concentration, using mock clusters with richness between $[32,34]$ in the BigMDPL simulation. Gray and red contours show the distributions of the average $3 \mathrm{D}$ and projected $2 \mathrm{D}$ membership distances, respectively, calculated using all galaxies within virial radius. Green and blue contours are for the average $2 \mathrm{D}$ membership distance $\left\langle R_{\mathrm{mem}}^{2 d}\right\rangle$ using galaxies projected within the redMaPPer aperture $R_{\lambda}$ and $0.65 R_{\lambda}$, respectively. Horizontal dashed lines indicate the median values of the four $\left\langle R_{\mathrm{mem}}\right\rangle \mathrm{vs}$. concentration distributions, while the vertical dashed line the median value of halo concentration. The correlation between $\left\langle R_{\text {mem }}\right\rangle$ and halo concentration becomes much weaker when projected distances are used and fewer galaxies are included, but still persists in the bottom blue contour where the definition of $\left\langle R_{\mathrm{mem}}\right\rangle$ closely resembles the $\left\langle R_{\text {mem }}^{\text {cut }}\right\rangle$ measurement in redMaPPer.

the $2 \mathrm{D}\left\langle R_{\text {mem }}\right\rangle$ estimator (red; $\rho_{c c}=-0.45$ ) is used, and weakens even further when extra aperture cuts of $R_{\lambda}$ (green; $\rho_{c c}=-0.42$ ) and $0.65 R_{\lambda}$ (blue; $\rho_{c c}=-0.31$ ) on $R$ are placed.

Fortunately, there is still substantial correlation left between $\left\langle R_{\text {mem }}^{\text {mock }}\right\rangle$ and $c$, and the large (small) $-\left\langle R_{\text {mem }}^{\text {mock }}\right\rangle$ subsample is still dominated by the intrinsically low (high)concentration clusters. In particular, the maximum assembly bias, as measured by the bias ratio between the low and high-concentration subsamples in the mock catalogue (using their 3D auto-correlation functions above $10 h^{-1} \mathrm{Mpc}$ ), is $1.24 \pm 0.02$, while the assembly bias retained by the $\left\langle R_{\text {mem }}^{\text {mock }}\right\rangle$ estimator is $60 \%$ weaker, i.e., $1.10 \pm 0.02$. However, we emphasize that this bias ratio of $1.10 \pm 0.02$ derived from using $\left\langle R_{\mathrm{mem}}^{\text {mock }}\right\rangle$ should be regarded as an upper limit of the observable signal in redMaPPer using $\left\langle R_{\mathrm{mem}}^{\text {cut }}\right\rangle$, because in the mock data the scatter between $\left\langle R_{\mathrm{mem}}\right\rangle$ and $c$ is under-estimated.

Expecting a bias ratio at most 1.10 based on mock data, 


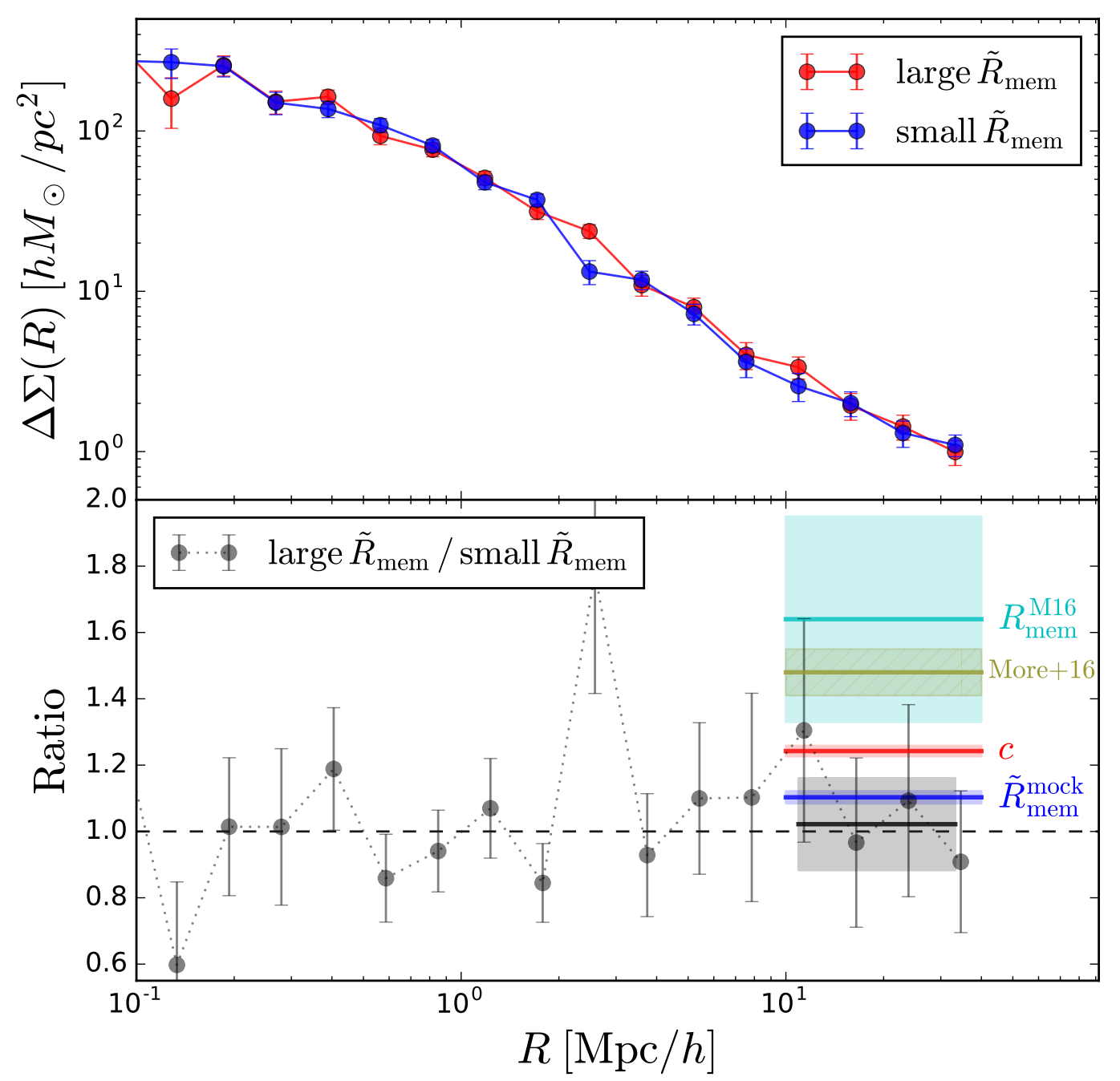

Figure 8. Stacked weak lensing measurements of the large- and small- $\left\langle R_{\mathrm{mem}}^{\text {cut }}\right\rangle$ cluster subsamples. The bottom panels show the ratios between the two profiles, and the gray band highlights the ratio between the two large-scale biases and its 1- $\sigma$ error, computed using scales above $10 h^{-1} \mathrm{Mpc}$. Red and blue thin bands indicate the two types of assembly bias signals predicted by $\Lambda$ CDM, using subsamples split by halo concentration $c$ and $\tilde{R}_{\text {mem }}^{\text {mock }}$, respectively. Cyan and yellow bands indicate the bias ratios derived by M16 and More et al. (2016), which are $1.5 \sigma$ and $3.5 \sigma$ higher than the maximum assembly bias signal (red band), respectively. The weak lensing measurement (gray band) is consistent with the assembly bias signal predicted from using $\tilde{R}_{\text {mem }}^{\text {mock }}$, i.e., the average projected membership distance of member galaxies within $0.65 R_{\lambda}$ in the BigMDPL mock cluster catalogue.

we are now ready to go back to the redMaPPer clusters, and examine whether the level of halo assembly bias revealed by $\left\langle R_{\mathrm{mem}}^{\text {cut }}\right\rangle$ in the SDSS data is consistent with this $\Lambda \mathrm{CDM}$ expectation. Following M16, we employ the stacked weak lensing of clusters (see Simet et al. 2016 for details) to examine the ratio between the large-scale clustering biases of the large and small- $\left\langle R_{\mathrm{mem}}^{\mathrm{cut}}\right\rangle$ subsamples.

Fig. 8 presents the key result of this paper. In the top panel, we compare the stacked weak lensing signals between the large (red) and small- $\left\langle R_{\mathrm{mem}}^{\text {cut }}\right\rangle$ (blue) subsamples of clusters on scales between 0.1 and $40 h^{-1} \mathrm{Mpc}$. The two weak lensing profiles are consistent with each other on all measured scales, indicating no discrepancy in either halo mass $\left(<1 h^{-1} \mathrm{Mpc}\right)$ or large-scale bias $\left(>10 h^{-1} \mathrm{Mpc}\right)$. The bottom panel shows the ratio between the weak lensing signals of the large and small- $\left\langle R_{\mathrm{mem}}^{\mathrm{cut}}\right\rangle$ clusters. The black horizontal line with gray band indicate the large-scale bias ratio and its 1$\sigma$ uncertainty $(1.02 \pm 0.14)$, using the weak lensing measurements above $10 h^{-1} \mathrm{Mpc}$. The observed ratio is consistent with the blue thin band $(1.10 \pm 0.02)$, which shows the expected levels of assembly bias derived from the large and small$\left\langle R_{\mathrm{mem}}^{\text {mock }}\right\rangle$ clusters in the mock catalogue. However, with large errorbars, the observed ratio is also consistent with unity, i.e., having no assembly bias. Also shown in the bottom panel are three bands indicating the maximum assembly bias if halo concentration is accessible (red), and the relatively high bias ratios measured in M16 (cyan) and More et al. (2016) (yellow). The strong discrepancies between these two measurements and the maximum ratio disappear almost entirely after we remove the projection effects from $\left\langle R_{\mathrm{mem}}\right\rangle$ by using $\left\langle R_{\text {mem }}^{\text {cut }}\right\rangle$.

Therefore, our re-analysis demonstrates that the level of 
cluster assembly bias in the SDSS redMaPPer catalogue is consistent with the prediction from $\Lambda \mathrm{CDM}$ structure formation theory. Although we do not detect halo assembly bias, we are only limited by the statistical uncertainties in the weak lensing measurements, rather than projection effects in the estimate of $\left\langle R_{\mathrm{mem}}\right\rangle$. This is very encouraging because, with enough statistics, the residual assembly bias signal can be robustly detected with little systematic uncertainties in ongoing and future cluster surveys.

\section{CONCLUSION}

Halo assembly bias is one of the most robust features of structure formation under $\Lambda \mathrm{CDM}$, but its signature in massive clusters could be affected by systematic uncertainties due to projection effects, as well as noisy measurements of halo concentrations $c$ using average membership distances $\left\langle R_{\text {mem }}\right\rangle$.

Using the public SDSS DR8 redMaPPer cluster catalogue, we developed a mark correlation statistic for diagnosing projection effects in $\left\langle R_{\mathrm{mem}}\right\rangle$, particularly the contamination of cluster membership probabilities $p_{m}$ by background galaxies. By examining the mark correlation of $\left\langle R_{\mathrm{mem}}^{\mathrm{M} 16}\right\rangle$, the $\left\langle R_{\mathrm{mem}}\right\rangle$ estimator proposed by Miyatake et al. (2016), we discovered that the $\left\langle R_{\mathrm{mem}}^{\mathrm{M} 16}\right\rangle$ values are significantly correlated between two clusters that are close to each other on the sky but well-separated in redshift. Therefore, the strong discrepancy between clustering biases of two cluster subsamples split by $\left\langle R_{\mathrm{mem}}^{\mathrm{M} 16}\right\rangle$, measured via weak lensing (Miyatake et al. 2016) or cross-correlation with photometric galaxy catalogues (More et al. 2016), can be largely attributed to the background contamination in $p_{m}$, rather than an anomalous signal of halo assembly bias.

To re-assess the level of cluster assembly bias in SDSS, we have developed a new variant of the $\left\langle R_{\text {mem }}\right\rangle$ estimator by excluding the low-membership probability galaxies $\left(p_{m}<0.8\right)$ that are strongly affected by the contamination in $p_{m}$. Further null test indicates that the new estimator $\left\langle R_{\mathrm{mem}}^{\text {cut }}\right\rangle$ is a clean indicator of galaxy concentration free of projection effects. In the longer term, however, it would be valuable to have a more robust method of assigning $p_{m}$ values that can be used directly with minimal projection effects on both cluster richness and $\left\langle R_{\mathrm{mem}}\right\rangle$.

In order to evaluate the impact of scatter between $\left\langle R_{\mathrm{mem}}^{\mathrm{cut}}\right\rangle$ and halo concentration $c$ on cluster assembly bias detection, we constructed mock catalogues of clusters and red-sequence galaxies from a large-volume $\Lambda \mathrm{CDM}$ simulation, and predicted that the bias ratio between the two cluster subsamples split by $\left\langle R_{\mathrm{mem}}^{\text {cut }}\right\rangle$ should be at most $1.10 \pm 0.02$, i.e., at least $60 \%$ weaker than the signal predicted using clusters split by halo concentration (1.24). This reduction is mainly caused by the combination of Poisson fluctuations in the galaxy number profile per cluster, and the small aperture size and projected distances used for defining $\left\langle R_{\mathrm{mem}}^{\mathrm{cut}}\right\rangle$.

From the weak lensing measurements of redMaPPer cluster subsamples split by $\left\langle R_{\mathrm{mem}}^{\text {cut }}\right\rangle$, we discovered that while having equal average masses, the bias ratio of the two subsamples is $1.02 \pm 0.14$, consistent with the prediction from our mock $\Lambda \mathrm{CDM}$ clusters as well as unity, i.e., having no assembly bias. Therefore, although no detection can be made due to large statistical uncertainties in the bias ratio, our result demonstrates that the level of halo assembly bias exhibited in the SDSS redMaPPer catalogue is consistent with $\Lambda \mathrm{CDM}$.

Within the present SDSS redMaPPer catalogue, a statistically significant detection of cluster assembly bias requires either significant reduction in the uncertainties of weak lensing measurements, or great improvement in the assignment of membership probabilities. However, with an order of magnitude increase in the observed cluster number counts, ongoing surveys like the Dark Energy Survey (The Dark Energy Survey Collaboration 2005) and the Hyper Suprime-Cam (Miyazaki et al. 2012) will deliver a smokinggun detection of halo assembly bias in the very near future.

\section{ACKNOWLEGEMENTS}

We thank Hironao Miyatake and Surhud More for carefully reading all previous versions of the manuscript and for giving detailed comments and suggestions that have greatly improved the manuscript. We also thank Bhuvnesh Jain, Neal Dalal, and Zheng Zheng for stimulating discussions at the early stage of this investigation, and David Spergel and Masahiro Takada for helpful comments on the final version of the manuscript. YZ and RM acknowledge the support by the U.S. Department of Energy (DOE) Early Career Program. YZ is also supported by a CCAPP fellowship. ER acknowledges support by the DOE Early Career Program, DOE grant DE-SC0015975, and the Sloan Foundation, grant FG-2016-6443. ESR is supported in part by the DOE contract to SLAC no. DE-AC02-76SF00515.

\section{REFERENCES}

Aihara H., et al., 2011, ApJS, 193, 29

Bardeen J. M., Bond J. R., Kaiser N., Szalay A. S., 1986, ApJ, 304, 15

Baxter E. J., Rozo E., Jain B., Rykoff E., Wechsler R. H., 2016, preprint, (arXiv:1604.00048)

Behroozi P. S., Wechsler R. H., Wu H.-Y., 2013, ApJ, 762, 109

Beisbart C., Kerscher M., 2000, ApJ, 545, 6

Berlind, A. A., Kazin, E., Blanton, M. R., et al. 2006, arXiv:astro$\mathrm{ph} / 0610524$

Blanton M. R., Berlind A. A., 2007, ApJ, 664, 791

Budzynski J. M., Koposov S. E., McCarthy I. G., McGee S. L., Belokurov V., 2012, MNRAS, 423, 104

Campbell D., van den Bosch F. C., Hearin A., Padmanabhan N., Berlind A., Mo H. J., Tinker J., Yang X., 2015, MNRAS, 452, 444

Castignani G., Benoist C., 2016, preprint, (arXiv:1606.08744)

Chen J., Kravtsov A. V., Prada F., Sheldon E. S., Klypin A. A., Blanton M. R., Brinkmann J., Thakar A. R., 2006, ApJ, 647, 86

Croton D. J., Gao L., White S. D. M., 2007, MNRAS, 374, 1303

Dalal N., 2016, Physics Online Journal, 9, 9

Dalal N., White M., Bond J. R., Shirokov A., 2008, ApJ, 687, 12

Deason A. J., Conroy C., Wetzel A. R., Tinker J. L., 2013, ApJ, 777,154

Diemand J., Moore B., Stadel J., 2004, MNRAS, 352, 535

Diemand J., Kuhlen M., Madau P., 2007, ApJ, 667, 859

Erickson B. M. S., Cunha C. E., Evrard A. E., 2011, Phys. Rev. D, 84, 103506

Gao L., White S. D. M., 2007, MNRAS, 377, L5 
Gao L., White S. D. M., Jenkins A., Stoehr F., Springel V., 2004, MNRAS, 355, 819

Gao L., Springel V., White S. D. M., 2005, MNRAS, 363, L66

Hahn O., Porciani C., Dekel A., Carollo C. M., 2009, MNRAS, 398,1742

Han J., Cole S., Frenk C. S., Jing Y., 2016, MNRAS, 457, 1208

Harker G., Cole S., Helly J., Frenk C., Jenkins A., 2006, MNRAS, 367, 1039

Jing Y. P., Suto Y., Mo H. J., 2007, ApJ, 657, 664

Kauffmann G., Li C., Zhang W., Weinmann S., 2013, MNRAS, 430, 1447

Klypin A., Yepes G., Gottlöber S., Prada F., Heß S., 2016, MNRAS, 457, 4340

Lacerna I., Padilla N., Stasyszyn F., 2014, MNRAS, 443, 3107

Lehmann B. V., Mao Y.-Y., Becker M. R., Skillman S. W., Wechsler R. H., 2015, preprint, (arXiv:1510.05651)

Li Y., Mo H. J., Gao L., 2008, MNRAS, 389, 1419

Lin Y.-T., Mandelbaum R., Huang Y.-H., Huang H.-J., Dalal N., Diemer B., Jian H.-Y., Kravtsov A., 2016, ApJ, 819, 119

Ludlow A. D., Navarro J. F., Springel V., Jenkins A., Frenk C. S., Helmi A., 2009, ApJ, 692, 931

McEwen J. E., Weinberg D. H., 2016, preprint, (arXiv: 1601.02693)

Medezinski E., Battaglia N., Coupon J., Cen R., Gaspari M., Strauss M. A., Spergel D. N., 2016, preprint, (arXiv: 1610.01624)

Miyatake H., More S., Takada M., Spergel D. N., Mandelbaum R., Rykoff E. S., Rozo E., 2016, Physical Review Letters, 116, 041301

Miyazaki S., et al., 2012, in Ground-based and Airborne Instrumentation for Astronomy IV. p. 84460Z, doi:10.1117/12.926844

More S., et al., 2016, ApJ, 825, 39

Nagai D., Kravtsov A. V., 2005, ApJ, 618, 557

Noh Y., Cohn J. D., 2012, MNRAS, 426, 1829

Paranjape A., Kovač K., Hartley W. G., Pahwa I., 2015, MNRAS, 454, 3030

Planck Collaboration et al., 2015, preprint, (arXiv:1502.01589)

Rykoff E. S., et al., 2014, ApJ, 785, 104

Sheth R. K., Tormen G., 1999, MNRAS, 308, 119

Sheth R. K., Tormen G., 2004, MNRAS, 350, 1385

Sheth, R. K., Connolly, A. J., \& Skibba, R. 2005, arXiv:astro$\mathrm{ph} / 0511773$

Simet M., McClintock T., Mandelbaum R., Rozo E., Rykoff E., Sheldon E., Wechsler R. H., 2016, preprint, (arXiv: 1603.06953)

Skibba R., Sheth R. K., Connolly A. J., Scranton R., 2006, MNRAS, 369, 68

Stoyan D., Stoyan H., 1994, Fractals, Random Shapes, and Point Fields: Methods of Geometrical Statistics. John Wiley \& Sons

Sunayama T., Hearin A. P., Padmanabhan N., Leauthaud A., 2016, MNRAS, 458, 1510

The Dark Energy Survey Collaboration 2005, arXiv:astro$\mathrm{ph} / 0510346$

Wang H., Mo H. J., Jing Y. P., 2009, MNRAS, 396, 2249

Wang L., Weinmann S. M., De Lucia G., Yang X., 2013, MNRAS, 433, 515

Wang W., Sales L. V., Henriques B. M. B., White S. D. M., 2014, MNRAS, 442, 1363

Wechsler R. H., Zentner A. R., Bullock J. S., Kravtsov A. V., Allgood B., 2006, ApJ, 652, 71

Weinmann S. M., van den Bosch F. C., Yang X., Mo H. J., 2006, MNRAS, 366, 2

Wu H.-Y., Rozo E., Wechsler R. H., 2008, ApJ, 688, 729

Yang X., Mo H. J., van den Bosch F. C., Weinmann S. M., Li C., Jing Y. P., 2005, MNRAS, 362, 711

Yang X., Mo H. J., van den Bosch F. C., 2006, ApJ, 638, L55
Yang X., Mo H. J., van den Bosch F. C., Pasquali A., Li C., Barden M., 2007, ApJ, 671, 153

York D. G., et al., 2000, AJ, 120, 1579

Zenteno A., et al., 2016, MNRAS, 462, 830

Zentner A. R., Hearin A. P., van den Bosch F. C., 2014, MNRAS, 443, 3044

Zentner A. R., Hearin A., van den Bosch F. C., Lange J. U., Villarreal A., 2016, preprint, (arXiv:1606.07817)

Zhu G., Zheng Z., Lin W. P., Jing Y. P., Kang X., Gao L., 2006, ApJ, 639, L5

Zu Y., Mandelbaum R., 2015, MNRAS, 454, 1161

Zu Y., Mandelbaum R., 2016, MNRAS, 457, 4360

Zu Y., Mandelbaum R., 2017, arXiv:1703.09219

Zu Y., Zheng Z., Zhu G., Jing Y. P., 2008, ApJ, 686, 41

van den Bosch F. C., Jiang F., Campbell D., Behroozi P., 2016, MNRAS, 455, 158

This paper has been typeset from a $\mathrm{T}_{\mathrm{E}} \mathrm{X} / \mathrm{LAT}_{\mathrm{E}} \mathrm{X}$ file prepared by the author. 